\title{
Modificações na quantidade e qualidade da radiação solar ao atravessar a atmosfera e interagir com plantas de soja
}

\author{
Denise Cybis Fontana ${ }^{1(*)}$, Genei Antonio Dalmago ${ }^{2}$, Juliano Schirmbeck ${ }^{3}$, Lucimara Wolfarth Schirmbeck ${ }^{4}$ \\ José Maurício Cunha Fernandes ${ }^{2}$ \\ ${ }^{1}$ Universidade Federal do Rio Grande do Sul - Faculdade de Agronomia. Avenida Bento Gonçalves 7712, Caixa Postal 15096, CEP 91501.970. Porto Alegre, \\ RS. E-mail: dfontana@ufrgs.br \\ ${ }^{2}$ Embrapa Trigo, Rodovia BR 285, km 294, Caixa Postal 3081, CEP 99050-970 Passo Fundo, RS. E-mail: genei.dalmago@embrapa.br e \\ mauricio.fernandes@embrapa.br \\ ${ }^{3}$ UNIVATES. Rua Avelino Talini, 171, Bairro Universitário, CEP 95914-014 Lajeado, RS. E-mail: schirmbeck.j@gmail.com \\ ${ }^{4}$ Universidade Federal do Rio Grande do Sul, Programa de Pós-Graduação em Sensoriamento Remoto - PPGSR, Centro Estadual de Pesquisa em Sensoria- \\ mento Remoto e Meteorologia - CEPSRM. Av. Bento Gonçalves, 9500 - Campus do Vale- Caixa Postal: 15044, CEP $91501-970$ Porto Alegre, RS. \\ E-mail: lucimaraws@gmail.com \\ ${ }^{(*)}$ Autor para correspondência.
}

\section{INFORMAÇÕES}

\section{História do artigo:}

Recebido em 11 de setembro de 2019

Aceito em 4 de março 2020

Termos para indexação:

radiação solar global

rRadiação Fotossinteticamente Ativa

reflectância

índices de vegetação
RESUMO

O objetivo deste trabalho foi caracterizar e dimensionar as modificações, quantitativas e qualitativas, na radiação solar no topo da atmosfera ao atravessar a atmosfera terrestre em dias ensolarados e após interação com superfície cultivada com soja. Os dados foram provenientes de experimento desenvolvido em uma lavoura de soja, município de Carazinho, RS, de novembro de 2017 a abril de 2018. Foram medidos parâmetros biofísicos, meteorológicos e radiométricos em dias ensolarados, caracterizando diferentes etapas de desenvolvimento no ciclo da soja. Foram analisados os dados de radiação no Topo de Atmosfera (Ra), radiação solar global (Rg), da Radiação Fotossinteticamente Ativa (PAR) e as medidas contínuas dos sensores espectrais da radiação incidente (i) e refletida (r) no espectro do vermelho (V) e do infravermelho próximo (IVP). A partir dos dados foram determinadas as razões $\mathrm{Rg} / \mathrm{Ra}$, PAR/Rg, Vi/VIPi e Vr/IVPr, assim como dois índices de vegetação. Os resultaram demonstraram que a atmosfera terrestre, assim como as condições da superfície, modifica a qualidade e quantidade da radiação solar que estará disponível para as plantas para seu crescimento e, consequentemente, podem diferenciar regiões de produção de alimentos.

(c) 2020 SBAgro. Todos os direitos reservados.

\section{Introdução}

Considerando o contexto em que a radiação solar é a fonte básica de energia para a produção de alimentos no planeta e de que a pressão por uma produção agrícola maior e de maior qualidade é crescente, surge a necessidade de aprofundar estudos para um melhor entendimento das interações da radiação com a atmosfera e com a superfície terrestre. Tal conhecimento pode indicar possibilidades de avanços no sentido de melhor aproveitamento 
deste recurso natural e ampliar os limites potenciais de produtividade.

O total diário de radiação solar recebido no topo da atmosfera ( $\mathrm{Ra}$ ) é um valor teórico importante e representa o potencial de energia incidente na região, sendo variável conforme a latitude e o dia do ano. Deste total, somente parte será absorvido pela superfície terrestre e aproveitado nos diversos processos biofísicos que nela ocorrem.

A radiação solar global (Rg) é uma medida do total diário de radiação que chega realmente à superfície, sendo depende das condições meteorológicas locais e do estado atmosférico. Ao atravessar a atmosfera, a radiação interage com seus constituintes resultando em modificação na quantidade, na qualidade e na direção dos raios solares. Essa interação ocorre de dois modos principais: absorção e espalhamento, os quais dependem do comprimento de onda da radiação e do tamanho do constituinte atmosférico. Há, portanto, uma interação seletiva que depende das condições atmosféricas do local (Pereira et al., 2002).

Do total de radiação solar que incide na superfície (0,3 a $4 \mu \mathrm{m})$, as faixas espectrais mais importantes para as plantas situam-se no espectro visível ( 0,4 a 0,7 $\mu \mathrm{m})$, o que é também considerado como sendo a radiação fotossinteticamente ativa (PAR), pois os pigmentos vegetais absorvem radiação dentro desta faixa de comprimento de ondas. Nesta, destacam-se as faixas entre 0,4 a 0,51 um (azul e violeta), que é fortemente absorvida pelos pigmentos verdes a amarelos das plantas, para fotossíntese, e efeitos formativos e a faixa

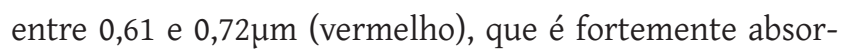
vida pelas clorofilas, para fotossíntese, e pelos fitocromos responsáveis por processos de fotoperiodismo. Fora do espectro visível, também muito importante é a faixa de 0,72 a 1,0 $\mu \mathrm{m}$ (infravermelho próximo), que também é necessária em processos de fotoperiodismo e alongamento de tecidos (Bergamaschi \& Bergonci, 2017). A quantificação das porções de Rg que incidem nas faixas apontadas acima como importantes para as plantas e, portanto, para a produção de alimentos, podem ser uteis na identificação de potencialidades ou limitações regionais e estacionais à obtenção de maiores rendimentos de grãos.

Também é relevante ressaltar o uso cada vez mais intenso de técnicas e produtos derivados do sensoriamento remoto no setor de produção de alimentos. Em especial, os sensores passivos que usam como fonte de energia a radiação solar incidente e fazem inferências sobre os distintos alvos na superfície a partir da forma como os mesmos interagem com a radiação. Neste setor, destacam-se o uso dos índices de vegetação, os quais possibilitam o monitoramento de biomassa verde das regiões de produção ao utilizar a relação antagônica da reflectância nas bandas do vermelho e do infravermelho próximo frente ao acúmulo de biomassa (Jensen, 2009).

Entre as aplicações mais frequentes dos índices de ve- getação estão as inferências sobre parâmetros biofísicos (Monteiro et al., 2012; Almeida, 2008), monitoramento e a modelagem do rendimento de grãos (Melo et al., 2008; Gleyce et al., 2016; Gusso et al., 2017) ou mesmo a indicação da sanidade das plantas, como doenças e deficiências nutricionais (Pinto et al., 2017).

Apesar do uso intenso dos índices de vegetação, algumas incertezas relacionas às condições de aquisição dos dados de reflectância precisam ser melhor compreendidas, de forma a contribuir para um entendimento dos seus valores, assim como dos diversos modelos ajustados e das limitações existentes.

O objetivo deste trabalho foi caracterizar e dimensionar as modificações, em termos quantitativos e qualitativos, que ocorrem na radiação solar que incide no topo da atmosfera ao atravessar a atmosfera terrestre em dias ensolarados e após sua interação com uma superfície cultivada com soja.

\section{Materiais e Métodos}

O experimento foi desenvolvido em área experimental sobre uma lavoura de soja de 28ha (Granja Capão Grande), localizada no município de Carazinho (lat 28¹3'43.89"S long 52 $54^{\prime} 15.93^{\prime \prime}$ ), em uma parceria entre a UFRGS e a EMBRAPA Trigo. O clima é Cfa, segundo a classificação de Köppen, subtropical úmido, com verões quentes e chuvas com distribuição regular ao longo do ano (Alvares et al., 2013). O Solo da área é um Latossolo Vermelho Aluminoférico típico (Streck et al., 2008).

A soja foi semeada em 13 de novembro de 2017 e a colheita em 03 de abril de 2018. A cultivar utilizada foi a DM 5958 RSF IPRO, semeada no espaçamento de $45 \mathrm{~cm}$ entre linhas e densidade de 24 plantas $\mathrm{m}^{-2}$. A adubação de base seguiu a análise de solo e correspondeu a $300 \mathrm{~kg} \mathrm{ha}^{-1}$ de NPK, na fórmula 4-28-08 na semeadura e uma aplicação $160 \mathrm{~kg}$ $\mathrm{ha}^{-1}$ de cloreto de potássio a lanço no dia anterior à semeadura. O controle de plantas daninhas, de pragas e de doenças foi realizado quando necessário, utilizando produtos indicados para a cultura (Reunião..., 2016) e foi baseado em monitoramento constante realizado no campo. Ao longo de todo este período foram medidos parâmetros biofísicos, meteorológicos e radiométricos.

Semanalmente, durante o ciclo da cultura foi registrado o estádio fenológico das plantas (Fehr \& Caviness, 1977). A cada semana foram colhidas quatro repetições de plantas, em um metro de linha, e as folhas foram separadas para determinação do índice de área foliar, a partir da área foliar específica. Para a determinação da área foliar específica quatro plantas, em sequência foram colhidas e das folhas foi determinada a área foliar de cada planta, com planímetro óptico (modelo 3100-L, marca LICOR) e determinada a matéria seca. A matéria seca das folhas de cada repetição 
e das quatro plantas individuais foi determinada em estufa de secagem de material vegetal a $65{ }^{\circ} \mathrm{C}$ e ventilação forçada. $\mathrm{O}$ ajuste matemático da função linear entre matéria seca de folhas e área foliar, gerou o coeficiente 175,02, que multiplicado à matéria seca de folhas das repetições, possibilitou estimar a área foliar de um metro de plantas. Esta área foliar foi dividida pela área de superfície correspondente $(0,45)$ estimando o índice de área foliar (IAF).

Do total de medições, foram escolhidos para análise 5 dias ensolarados, caracterizando diferentes etapas de desenvolvimento ao longo do ciclo da soja (Figura 1), desde os estádios V4 (Quarto trifólio aberto) ao R7.2 (Maturação fisiológica, com $50 \%$ a $75 \%$ folhas amarelas), segundo escala de Ferr \& Caviness (1977). Para estes dias foram analisados os dados de radiação no Topo de Atmosfera ( $\mathrm{Ra}$ - estimado pela equação de Planck, Eq. 1) e os dados de radiação solar global (Rg), medida com sensor modelo SP-110-L, marca Apogee, e da Radiação Fotossinteticamente Ativa (PAR), medida com sensor modelo SQ-110, marca Apogee, ambos conectados a datalogger modelo CR1000 da marca Campbell. Foram registradas ainda as medidas com sensores espectrais da radiação incidente (DECAGON SRS-NDVI Hemisférico) e refletida (DECAGON SRS NDVI com Limitador de Visão) no espectro do vermelho - V (0,64 a 0,66 $\mu \mathrm{m})$ e do infravermelho próximo - IVP $(0,81$ a $0,83 \mu \mathrm{m})$, conforme identificado na Figura 2. Todos os sensores foram conectados a registradores de dados com frequência de registro de $15 \mathrm{~min}$.

$$
R a=S\lceil\bar{d} / d\rceil^{2} \cos Z_{h}
$$

onde S é a constante solar; $\lceil\bar{d} / d\rceil$ é a razão entre a distância média e real do Sol a Terra e $Z_{h}$ é o ângulo de incidência dos raios solares.
Devido a problemas ocorridos no sensor instalado na área experimental, o dado de Rg foi medido somente nos dois primeiros dias de avaliações. Para os demais, Rg foi estimado a partir dos dados de PAR medidos, através da equação de regressão linear ajustada entre estes dois conjuntos de dados nestes dois primeiros dias em que houve medidas simultâneas.

Os sensores espectrais foram instalados em um mastro no centro da lavoura, a uma altura, ajustável ao longo do ciclo, de $1 \mathrm{~m}$ acima do topo do dossel (Figura 3). A partir destes dados foram determinadas a porção de radiação solar perdida ao atravessar a atmosfera (Rg/Ra), a porção de radiação solar que é fotossinteticamente ativa para as plantas (PAR/Rg), a proporção da radiação incidente e refletida nos espectros do Vermelho e infravermelho próximo (Vi/VIPi e Vr/IVPr).

Para caracterizar a interação da radiação solar com a superfície, foram calculadas as reflectâncias nos espectros V e IVP, obtidas pela razão entre a radiação refletida (sensor voltado para baixo) e incidente (sensor voltado para cima), usando as expressões: $\rho \mathrm{V}=\mathrm{Vr} / \mathrm{Vi}$ e $\rho I V P=I V P r / I V P i$.

Por fim, a partir destes dados de reflectâncias nos espectros V $\left(\rho_{\mathrm{V}}\right)$ e IVP $\left(\rho_{\text {IVP }}\right)$ obteve-se os índices de vegetação razão simples (SR) e índice de vegetação por diferença normalizada (NDVI) para a cultura da soja nos diferentes estádios de desenvolvimento, respectivamente usando as equações 2 e 3, e mostradas a variabilidade ao longo dos dias em análise.

$$
S R=\rho_{I V} / \rho_{V}
$$

$$
N D V I=\frac{\left(\rho_{I V}-\rho_{V}\right)}{\left(\rho_{I V}+\rho_{V}\right)}
$$

Figura 1: Índice de Área Foliar (IAF) ao longo do ciclo da soja expresso em Dias Após a Emergência (DAE). As letras no topo da figura são relativas aos estádios fenológicos sengundo escala de Ferr \& Caviness (1977).

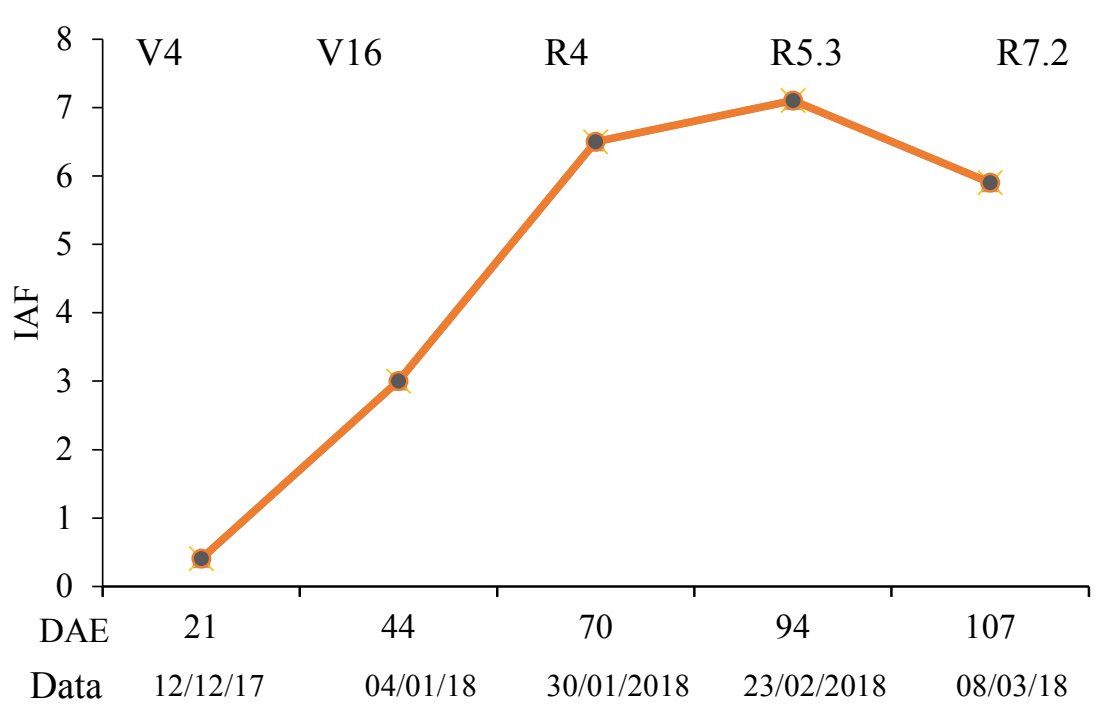

Agrometeoros, Passo Fundo, v.27, n.1, p.101-110, set 2019. 103 
Figura 2: Representação gráfica da lei de Planck para um corpo negro a $6.000 \mathrm{~K}$ com a identificação dos intervalos de comprimento de ondas medidos e avaliados no estudo (Rg - Radiação Solar Global, 0,4 a

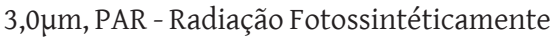

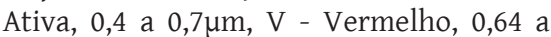
$0,66 \mu \mathrm{m}, \mathrm{IVP}$ - Infravermelho Próximo, 0,81 a $0,83 \mu \mathrm{m}$

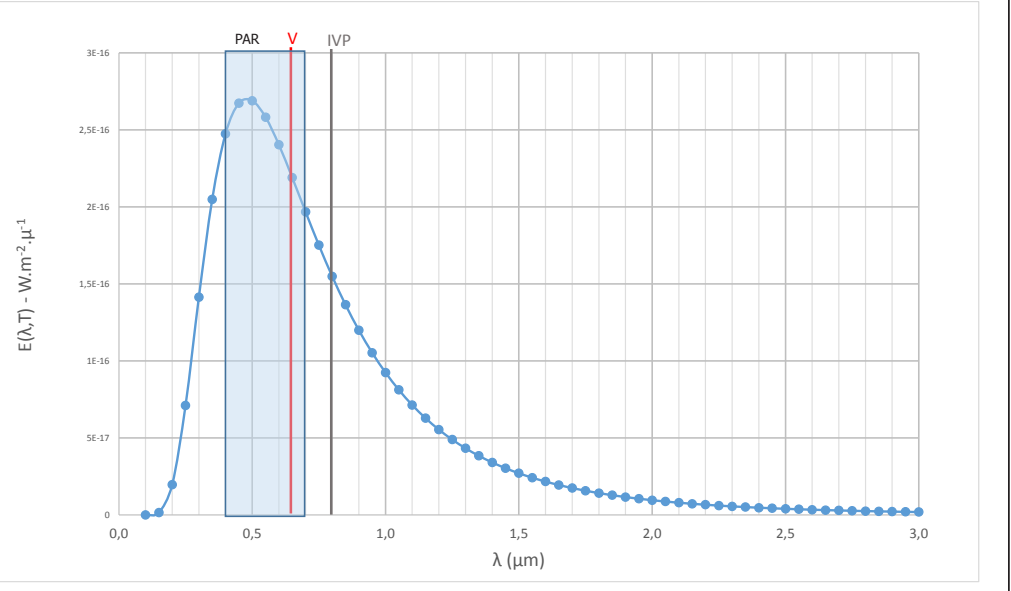

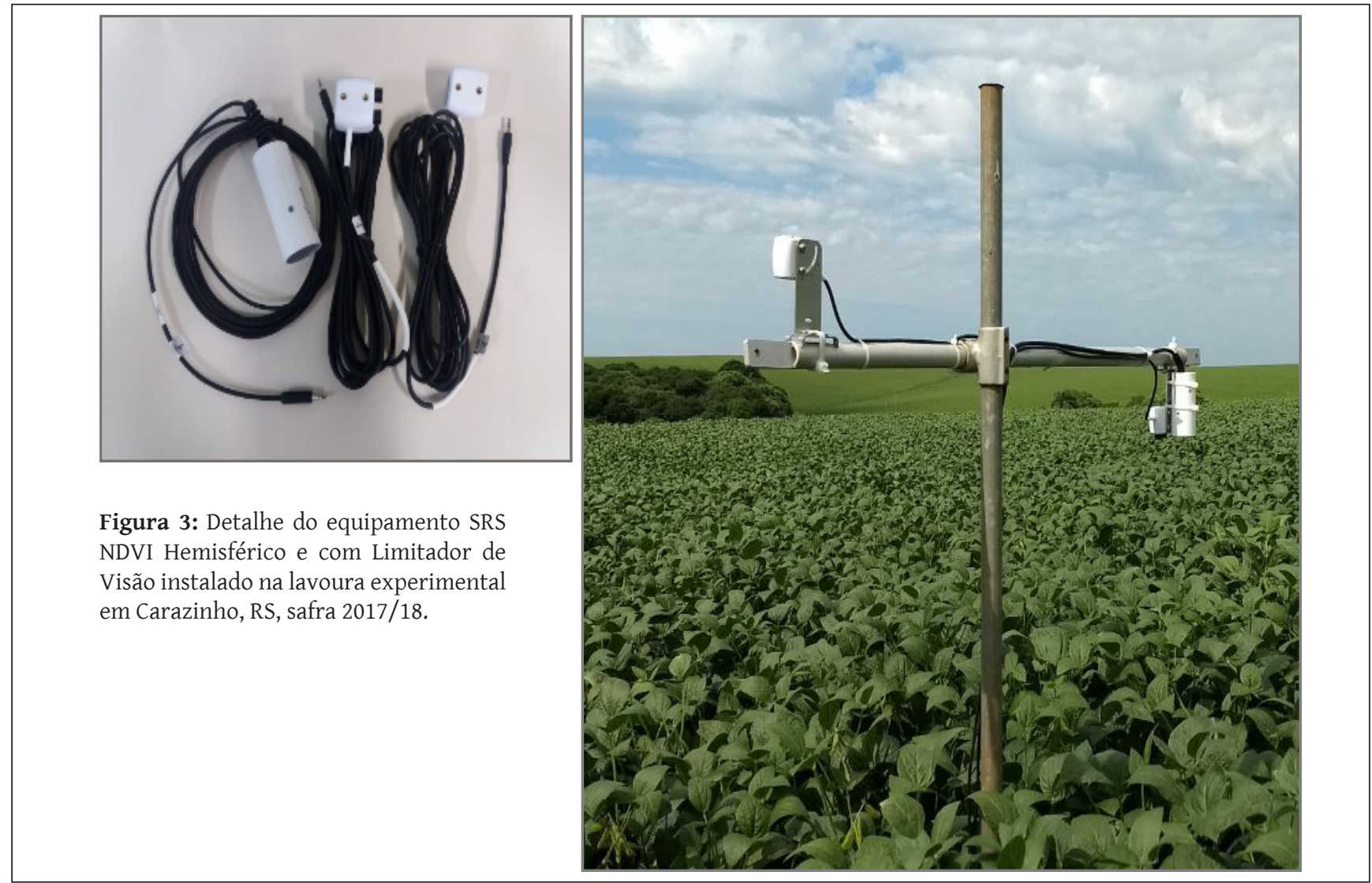

\section{Resultados e Discussão}

Conforme esperado, Rg e PAR apresentam uma relação linear, com baixa dispersão, em que incrementos em Rg resultaram em incrementos proporcionais em PAR. Em torno de 43,58\% de Rg corresponde a radiação fotossinteticamente ativa (Figura 4), proporção similar a obtida em estudos correlatos (França et al., 1997; Martonaro, 2007).

O curso diário, tanto de Rg como de PAR de todos os dias de medição (Figura 5) evidencia que mesmo tendo sido selecionado dias ensolarados para as análises, em alguns horários houve pequenas reduções no fluxo incidente associadas à ocorrência de nuvens esparsas. Neste estudo, a influência das nuvens na redução do fluxo de radiação incidente não foi considerada.
Os valores máximos de Rg ocorreram próximo às $12 \mathrm{~h}$. As diferenças de duração do dia e de densidade de fluxo de radiação solar foram decorrentes da geometria de iluminação Sol-Terra, a qual variou ao longo do período das medições. Para Rg, a densidade de fluxo de radiação incidente oscilou de 32,00 a 26,33 MJ m² dia $^{-1}$ (Tabela 1), sendo decrescente de dezembro de 2017 (solstício de verão) a março de 2018 (equinócio), conforme esperado.

A razão Rg/Ra, também conhecida como índice de claridade, representa uma proporção média de perdas na radiação associadas à atenuação atmosférica causadas pelos diversos fenômenos que ocorrem com a radiação que atravessa as camadas atmosféricas (absorção, reflexão e espalhamento). O valor obtido no presente estudo foi de 0,725, 
Figura 4: Gráfico de dispersão entre os dados de Radiação Solar Global (Rg) e Radiação Fotossinteticamente Ativa (PAR), ambos medidos na lavoura experimental em Carazinho, RS, safra 2017/18.

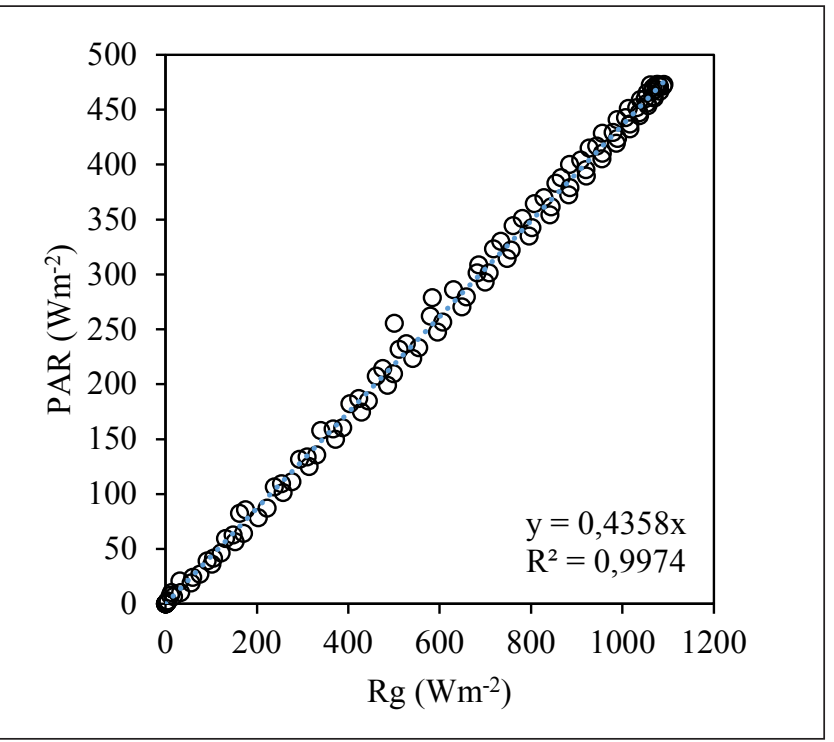

com pequena variação entre os 5 dias avaliados $(0,684$ a 0,739). Importante frisar que esta razão costuma assumir valores mais baixos do que os encontrados no presente estudo. No Rio Grande do Sul, Weber (2011), analisando dados da Serra Gaúcha, obteve valores oscilando de 0,345 a 0,534, com variações associadas à época do ano e localização geográfica. Marques et al. (2000), analisando dados de Pelotas encontrou variação entre 0,33 e 0,61. A diferença verificada, apesar de grande, mostra coerência, visto que, conforme já declarado, a presente análise se restringiu a dias ensolarados e, portanto, não considerou a importante interferência da nebulosidade na redução da radiação solar ao atravessar a atmosfera terrestre.

A análise da qualidade da radiação solar incidente, mostrou que a densidade de fluxo de radiação no espectro $V$ é, em média, 30\% maior do que o fluxo incidente no espectro IVP (Figura 6 A, B, C, D, E). A proporção Vi/IVPi é menor no início da manhã e final da tarde e se mantém estável nas horas centrais do dia (Figura 7). Neste período

Figura 5: Transcurso diário da Radiação Solar Global (Rg) e da Radiação Fotossinteticamente ativa (PAR) nos dias em análise, , Carazinho, RS, safra 2017/18.

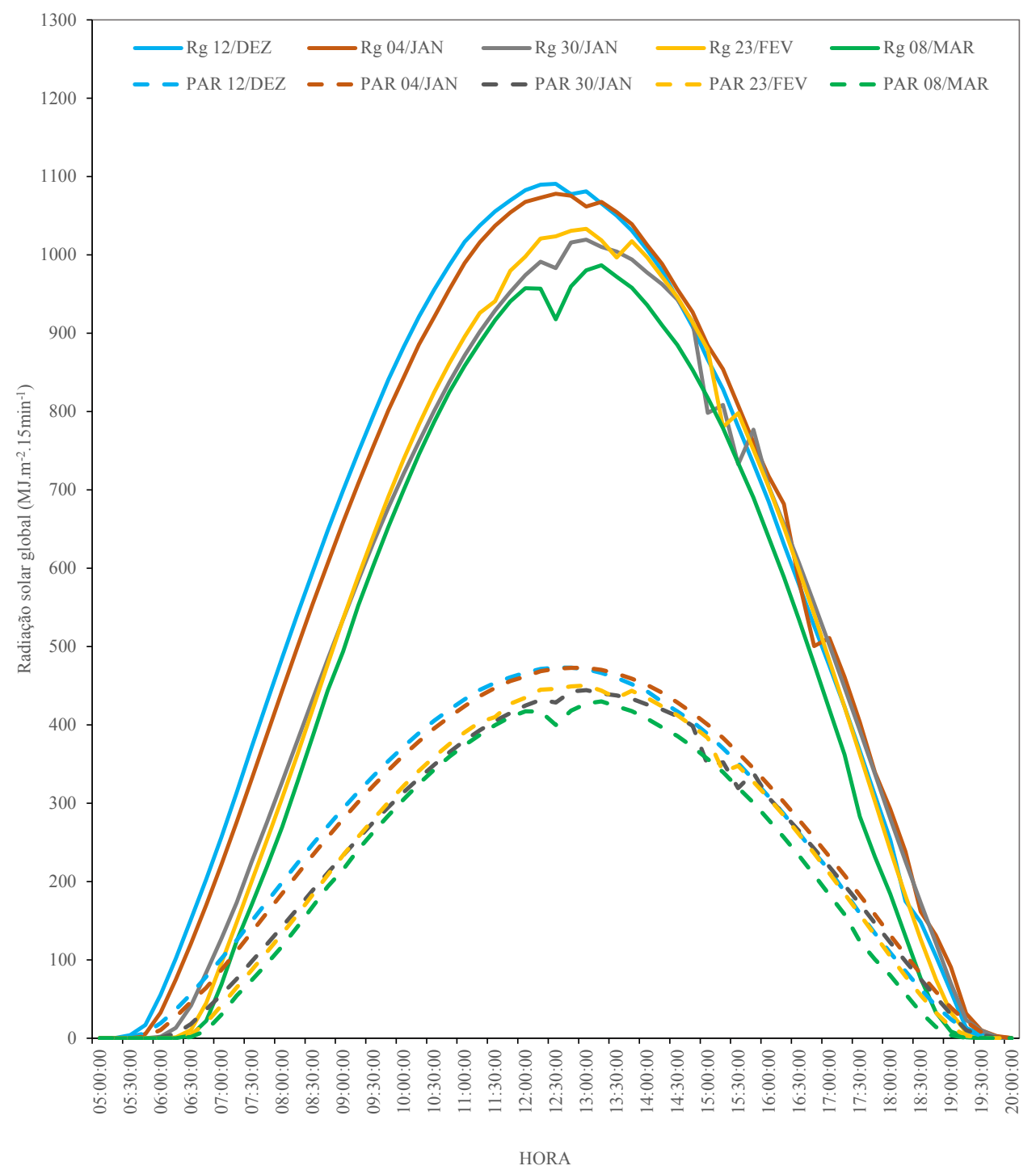


Figura 6: Transcurso diário do fluxo de radiação incidente (A, B. C, D e E) e refletida (F, $\mathrm{G}, \mathrm{H}, \mathrm{I}$ e J) no espectro do Vermelho (V) e do Infravermelho (IV), Carazinho, RS, safra 2017/18.
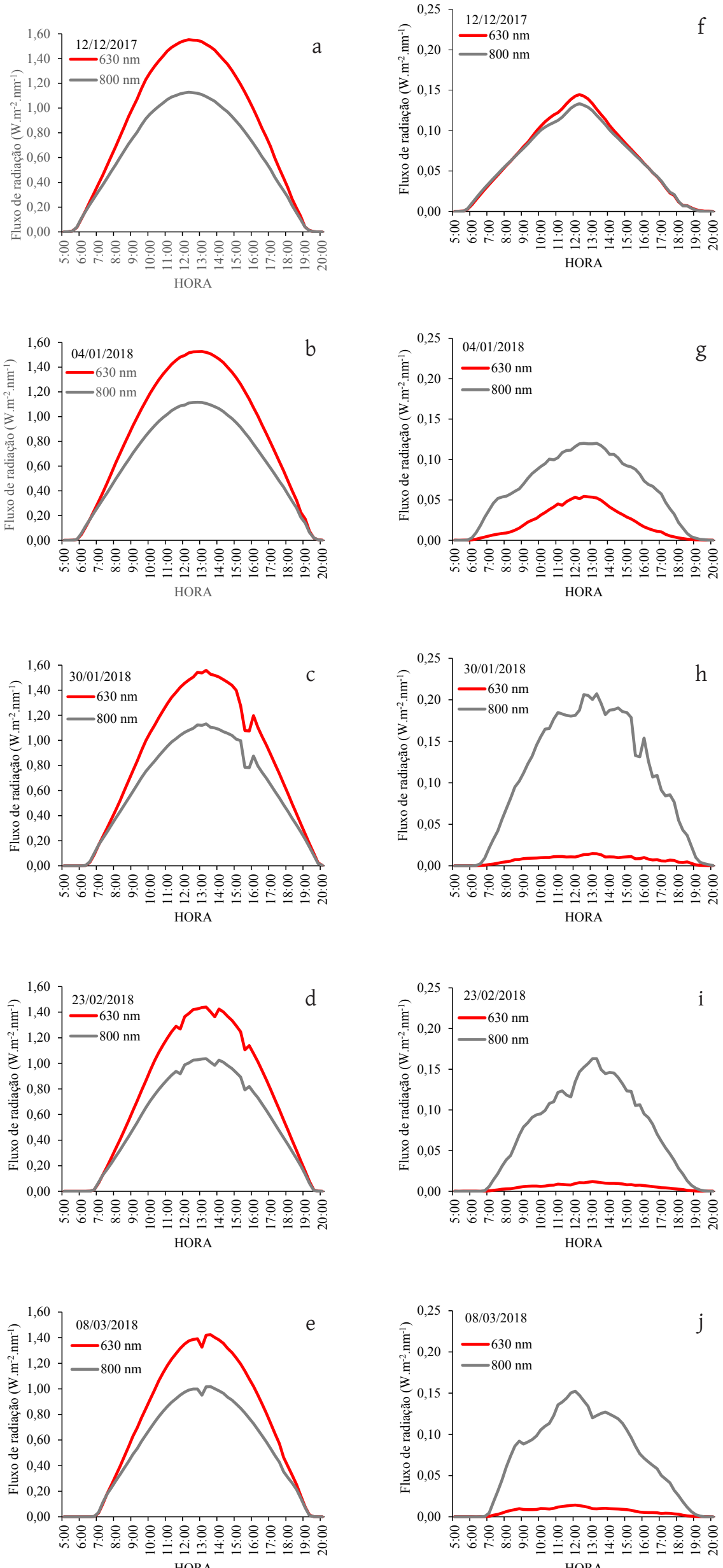
Tabela 1: Totais diários de Radiação Solar Global (Rg) e Radiação Fotossinteticamente ativa (PAR) nos dias em análise após a semeadura (DAS) e sua proporção em relação a Radiação Solar incidente no topo da Atmosfera (Ra), Carazinho, RS, safra 2017/18.

\begin{tabular}{|c|c|c|c|c|c|c|}
\hline DATA & DAS & $\begin{array}{c}\mathrm{Ra} \\
\mathrm{MJ} \cdot \mathrm{m}^{-2} \cdot \mathrm{dia}^{-2}\end{array}$ & $\begin{array}{c}\mathbf{R g} \\
\text { MJ.m } \mathbf{m}^{-2} \cdot \mathrm{dia}^{-1}\end{array}$ & $\begin{array}{c}\text { PAR } \\
\text { MJ.m }{ }^{-2} \cdot \mathrm{dia}^{-1}\end{array}$ & $\mathbf{R g} / \mathbf{R a}$ & PAR/Rg \\
\hline $12 / 12 / 2017$ & 21 & 43,70 & 32,00 & 13,82 & 0,732 & 0,432 \\
\hline $04 / 01 / 2018$ & 44 & 43,71 & 31,69 & 13,90 & 0,725 & 0,439 \\
\hline $30 / 01 / 2018$ & 70 & 41,79 & 28,60 & 12,47 & 0,684 & 0,436 \\
\hline $23 / 02 / 2018$ & 94 & 38,21 & 28,47 & 12,41 & 0,745 & 0,436 \\
\hline $08 / 03 / 2018$ & 107 & 35,63 & 26,33 & 11,48 & 0,739 & 0,436 \\
\hline
\end{tabular}

Figura 7: Transcurso diário da razão entre o fluxo de radiação incidente (A) e refletido (B) no espectro do Vermelho (V) e do Infravermelho (IV), Carazinho, RS, safra 2017/18.

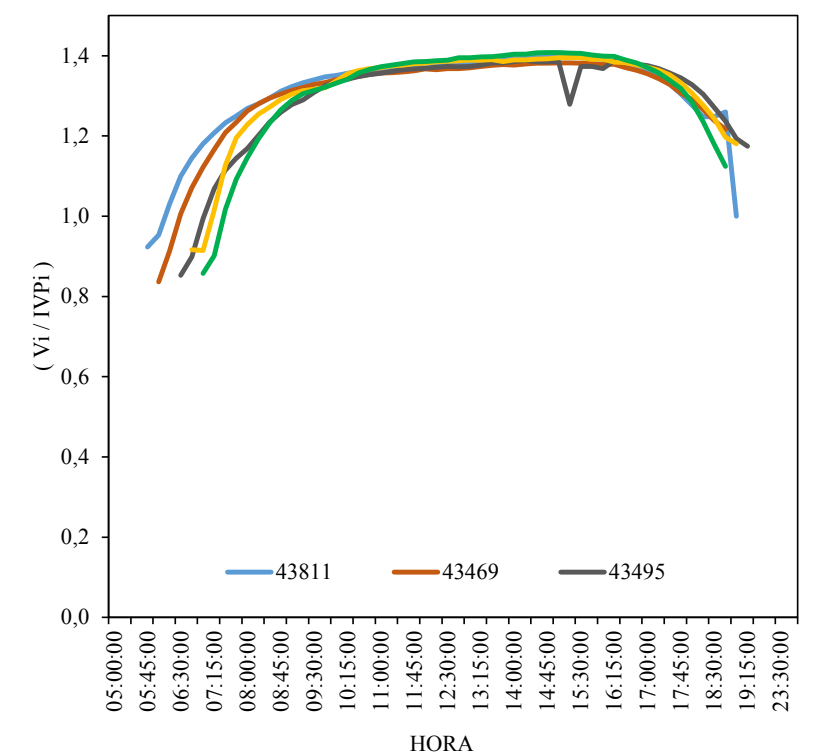

os valores foram próximos a 1,35, com pequenas diferenças ao longo dos 5 dias analisados.

Já quando se avaliou o espectro de radiação refletida, verifica-se um padrão diverso e mais variável. A densidade de fluxo de radiação refletida no IV foi em geral maior do que no $\mathrm{V}$ (Figura $6 \mathrm{G}, \mathrm{H}$, I e J) ao longo de todo o dia, com exceção do dia 12/12 (Figura 6 F). Os valores da razão Vr/ IVPr além de diferir quanto ao valor, também foram distintos entre os dias de medição (Figura 6 e 7), o que é explicado pelas alterações que ocorreram na lavoura de soja durante o período de medições, associadas ao crescimento e desenvolvimento da cultura (Figura 1), modificando a interação com a radiação solar incidente.

No dia $12 / 12$, com IAF de 0,4 , a resposta observada foi muito marcada pela interação da radiação solar com o solo. Como a radiação refletida no V e IVP são tipicamente similares nos solos (JENSEN, 2009), o resultado foram proporções Vr/IVPr próximas de 1,0. Nas datas subsequentes, a interação da radiação na superfície passou a ser modulada pela presença crescente de vegetação. Por um lado, a ab-

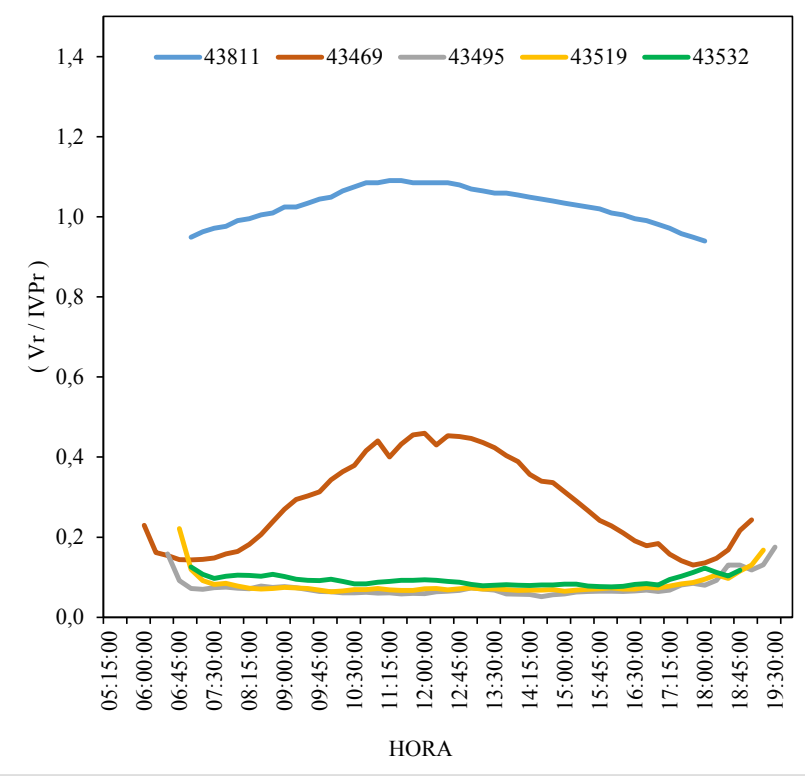

sorção da radiação V pelos pigmentos foliares (Ponsoni \& Shimabukuro, 2017) determinou uma redução na radiação refletida. Por outro lado, o espalhamento que ocorre no percurso da radiação IVP no mesófilo das folhas (Jensen, 2009), gerou o incremento na reflexão, resultando em razões Vr/IVPr inferiores a 0,4.

Em função da variação da densidade de fluxo incidente entre os dias de medição, é mais comum e conveniente para fins de comparações entre tipos de cobertura do solo ou para avaliar as diferenças de uma mesma cobertura ao longo do tempo, o uso de dados de radiação expressos na forma de reflectância ou de índices de vegetação (Jensen, 2009). A Figura 8 mostra o padrão observado nos 5 dias de medição para os índices SR e NDVI, evidenciando as diferenças entre eles.

SR é um índice obtido pela razão entre as reflectâncias IV e V, o que gera valores positivos e crescentes a medida em que ocorrem incrementos na biomassa verde presente na superfície. Para a soja, SR assumiu valores próximos a zero no primeiro dia de medição, onde a contribuição 
Figura 8: Transcurso diário dos índices SR (Razão simples) e NDVI (índice de vegetação por diferença normalizada) ao longo do ciclo da soja. Carazinho, RS, safra 2017/18.

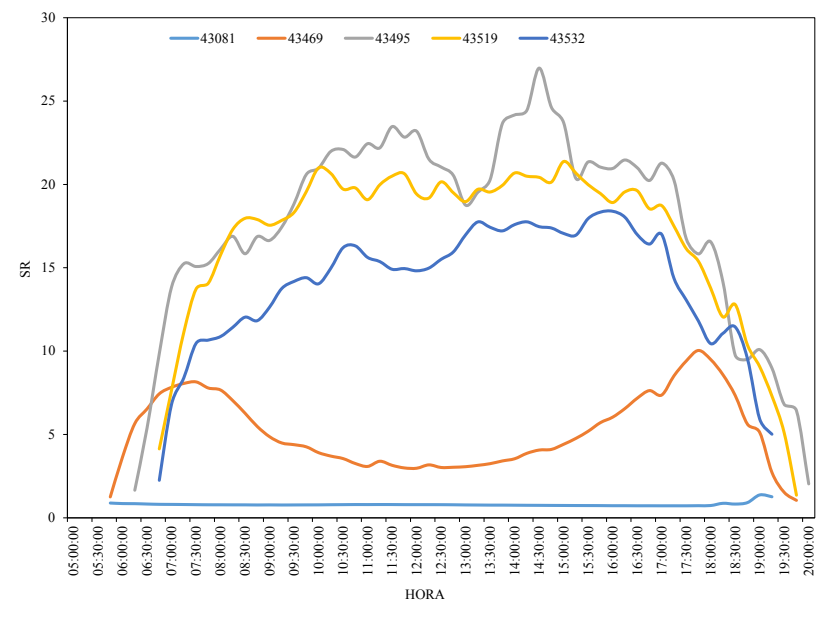

do solo era significativa, atingindo os valores máximos $(\approx$ 20) quando o IAF foi acima de 6,5 nos estádios R4 e R5.3. Observou-se um pequeno decréscimo ao final do ciclo, no estádio R7.2, quando já havia a presença de folhas amarelas, o que gerou incremento da reflexão no vermelho pela redução da ação dos pigmentos e uma redução na reflexão do IV em função do colapso das estruturas foliares. Valores similares deste índice para a cultura da soja foram obtidos por Almeida (2008) e Monteiro et al. (2012).

Já o NDVI é obtido pela diferença entre as reflectâncias no IVP e V, normalizado pela soma destas. A normalização determina que o intervalo possível de valores do índice oscile entre menos um e um (-1 e 1). Coberturas vegetais apresentam valores positivos de NDVI, dada a resposta antagônica nas porções V e IVP frente ao acúmulo de biomassa, o que é uma característica típica e única deste tipo de cobertura (Ponsoni \& Shimabukuro, 2017). Ao longo dos dias de medição, o NDVI da soja, a semelhança do observado na SR, também foi crescente com o crescimento da biomassa verde. Entretanto, o NDVI foi similar nos dias 30jan, 23-fev 8-mar, evidenciando uma das mais importantes limitações no uso do NDVI, que é a saturação do índice (Jensen, 2009; Ponsoni \& Shimabukuro, 2017). Aumentos ou reduções no IAF não resultam em alteração no NDVI, o que indica falta de sensibilidade do índice em altas densidades de biomassa. Fontana et al. (2019) identificou que a saturação do NDVI na lavoura de soja ocorre com IAF acima de 5, já Almeida (2008) observou a saturação com IAF um pouco mais baixo, 4,5 .

Ao longo do dia, o NDVI apresentou valores menores no início da manhã e final da tarde, quando o fluxo de radiação solar é bastante baixo e incide com maior inclinação. Após, ocorreu estabilização do NDVI, o que foi observado tanto no início como no final do ciclo da cultura.

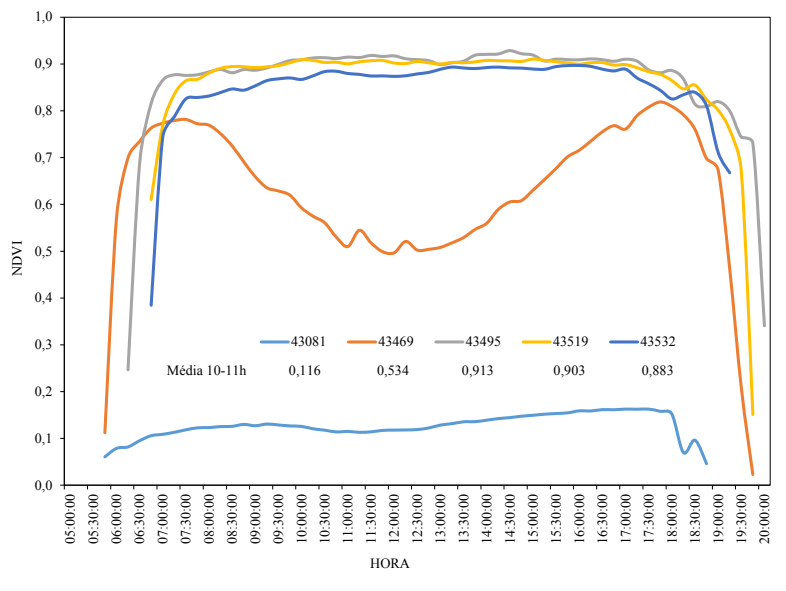

Em ambos os índices, SR e NDVI, nas medições tomadas no dia 4-janeiro, quando a cultura ainda não apresentava cobertura total do solo (IAF $=3,0$ ), verificou-se uma redução nos valores nos horários centrais do dia. As reduções possivelmente estejam associadas à influência do "fundo" na reflectância do dossel semeada em linhas, visto que palha ou solo descoberto tendem a reduzir os índices (Jensen, 2009). Esta contribuição maior do "fundo" em baixo IAF, também pode ser decorrente de mudança de inclinação das folhas e/ou efeito heliotrópico em função da alta demanda hídrica da atmosfera e/ou redução da disponibilidade de água no solo. Estes são fatores que devem ser ainda melhor estudados. Este resultado, com aspecto côncavo, já é conhecido (SRS, 2017) mas explicita a influência da geometria de aquisição dos dados (sol-sensor-superfície) e, portanto, da necessidade de padronização do horário de obtenção das medidas dos índices de vegetação, quando se deseja fazer comparações.

\section{Conclusão}

A atmosfera terrestre, assim como as condições da superfície, modifica a qualidade e quantidade da radiação solar que estará disponível para plantas realizarem o seu crescimento e, consequentemente, podem diferenciar regiões de produção de alimentos.

No noroeste do Rio Grande do Sul, em dias ensolarados, cerca de $70 \%$ da radiação que incide no topo da atmosfera atinge a superfície após a interação com os constituintes atmosféricos. Deste total, cerca de $43 \%$ representa a radiação que as plantas absorvem para o processo fotossintético.

A radiação que incide na superfície possui, em média, $35 \%$ mais fluxo nos comprimentos de onda do vermelho 
do que do infravermelho próximo. Após a interação com a superfície, esta proporção é modificada, mostrando dependência das condições de cobertura da superfície, aumentando a porção do infravermelho próximo à medida que ocorre aumento da cobertura de biomassa verde.

Os dois índices de vegetação (SR e NDVI) testados, que se baseiam na interação das plantas nos comprimentos de ondas do vermelho e infravermelho próximo, são capazes de diferenciar as condições de cobertura das plantas ao longo do ciclo da soja.

\section{Agradecimentos}

UFRGS e EMBRAPA Trigo agradecem aos proprietários da Fazenda Granja Capão Grande pela cedência da área experimental e ao CNPq pelo apoio financeiro para compra dos equipamentos e bolsa de Produtividade em Pesquisa. Os autores agradecem ainda aos funcionários da Embrapa Trigo, Elisson S. S. Pauletti e Cristian M. Plentz, que auxiliaram na condução do experimento e coletas dos dados. Parte do trabalho foi financiado pelo projeto Embrapa SEG 02.15.07.003.00.00.

\section{Referências}

ALMEIDA, T. Respostas espectrais da soja sob diferentes condições hídricas e de preparo de solo. Dissertação (PPG-Sensoriamento Remoto UFRGS), 2008.

ALVARES, C.A.; STAPE, J.L.; SENTELHAS, P.C.; GONÇALVES, J.L.M.; SPAROVEK, G. Köppen's climate classification map for Brazil. Meteorologische Zeitschrift 22:711-728. 2013.

FEHR, W. R.; CAVINESS, C. E. Stages of soybean development. Ames: Iowa State University of Science and Technology, 1977. 15p. (Special Report, 80).

FIGUEIREDO, G.K.D.A.; BRUNSELL, N.A.; ROCHA, J.V.; LAMPARELLI, R.A.C.; PICOLI, M.C.A. Using temporal stability to estimate soya bean yield: a case study in Paraná state, Brazil, International Journal of Remote Sensing, 37:5, 1223-1242, 2016.
FONTANA, D.C., SANTOS, L.N.; DALMAGO, G.; SCHIRMBECK, J.; SCHIRMBECK, L. NDVI e alguns fatores de variabilidade. In: SIMPÓSIO BRASILEIRO DE SENSORIAMENTO REMOTO, XIX, 2019. Santos, Anais... Disponível em http://sbsr.com.br/

FRANÇA, S. et ROSA, L.M.G. ; BERGAMASCHI, H. ; NABINGER, C. ; SPANENBERG, P. Radiação fotossinteticamente ativa e sua relação com a radiação solar global em dossel de alfafa em função do índice de área foliar. Revista Brasileira de Agrometeorologia, Santa maria, v.5, n.2, p.147-153, 1997.

GUSSO, A.; ARVOR, D.; DUCATI, J.R. Model for soybean production forecast based on prevailing physical conditions. Pesquisa Agropecuária Brasileira, v. 52, p. 95-103, 2017.

JENSEN, J.R. Sensoriamento Remoto do Ambiente: uma prespectiva em recursos terrestres. Tradução Epiphanio, J.C.N. Parênteses, São José dos Campos. 2009.

MARQUES, K., PEREIRA, T.P.; ASSIS, S.V. Análise do comportamento mensal do índice de limpidez. In: CONGRESSO BRASLEIRO DE METEOROLOGIA, XI, 2000, Rio de Janeiro, Anais... Rio de Janeiro, 2000. Disponível em

MARTORANO, L.G. Padrões de resposta da soja a condições hídricas do sistema solo-planta-atmosfera, observados no campo e simulados no sistema de suporte à decisão DSSAT. 2007. 151p. Tese (Doutorado) - Programa de Pós-Graduação em Fitotecnia, Faculdade de Agronomia, Universidade Federal do Rio Grande do Sul, Porto Alegre, 2007.

MELO, R.W.; FONTANA, D.D.; BERLATO, M.A.; DUCATI, J.R. An agrometeorological-spectral model to estimate soybean yield, applied to southern Brazil. International Journal of Remote Sensing, v.29, n.14, p.40134028, 2008.

MONTEIRO, P.F.C.; ANGULO FILHO, R.; XAVIER, A.C.; MONTEIRO, R.O.C. Assessing biophysical variable parameters of bean crop with hyperspectral measurements, Scientia Agricola v.69: p.87-94, 2012.

PINTO, D.G, FONTANA, D.C.; DALMAGO G.A; FOCHESATTO, E.; VICARI, M.B.; BREMM, C.; CUNHA, G.R.;GOUVÊA, J.A; SANTI, A. Correlations between spectral and biophysical data obtained in canola canopy cultivated in the subtropical region of Brazil. Pesquisa Agropecuária Brasileira, Brasília, v.52, n.10, p.825-832, 2017.

PONSONI, F.J.; SHIMABUKUTO, Y.E. Sensoriamento Remoto no estudo da Vegetação. Editora Parêntese, São José dos Campos, 127p., 2017.

Reunião de Pesquisa de Soja da Região Sul. Indicações técnicas para a cultura da soja no Rio Grande do Sul e em Santa Catarina, safras 2016/2017 e 2017/2018. [Organizadores] José Roberto Salvadori ... et al. - Passo Fundo : Ed. Universidade de Passo Fundo, 2016. 128p.

SRS Spectral Reflectance Sensor. Operator's Manual. Pullman WA. 2017. STRECK, E.V.; KÄMPF, N.; DALMOLIN, R. S. D.; KLAMT, E.; NASCIMENTO, P. C.; SCHNEIDER, P.; GIASSON, E.; PINTO L. F. S. Solos do Rio Grande do Sul. 2 ed. Porto Alegre: Emater/RS, 2008. 222 p.

\section{REFERENCIAÇÃO $\quad$ FONTANA, D. C.; DALMAGO, G. A.; SCHIRMBECK, J.; SCHIRMBECK, L. W.; FERNANDES, J. M. C. Modificações na quantidade e qualidade da radiação solar ao atravessar a atmosfera e interagir com \\ plantas de soja. Agrometeoros, Passo Fundo, v.27, n.1, p.101-110, set 2019.}

Declaração: os trabalhos estão sendo publicados nesse número de AGROMETEOROS (v.27, n.1, set 2019) conforme foram aceitos pelo XXI Congresso Brasileiro de Agrometeorologia, realizado de 12 a 16 de agosto de 2019, em Catalão, Goiás, sem revisão editorial adicional da revista. 


\title{
Modifications on the quantity and quality of solar radiation by crossing the atmosphere and interacting with soybean plants
}

\author{
Denise Cybis Fontana ${ }^{1(*)}$, Genei Antonio Dalmago ${ }^{2}$, Juliano Schirmbeck ${ }^{3}$, Lucimara Wolfarth Schirmbeck ${ }^{4}$ and \\ José Maurício Cunha Fernandes ${ }^{2}$
}

${ }_{1}^{1}$ Universidade Federal do Rio Grande do Sul - Faculdade de Agronomia. Avenida Bento Gonçalves 7712, Caixa Postal 15096, CEP 91501.970. Porto Alegre, RS, Brazil. E-mail: dfontana@ufrgs.br

${ }^{2}$ Embrapa Trigo. Rodovia BR 285, km 294, Caixa Postal 3081, CEP 99050-970 Passo Fundo, RS, Brazil. E-mail: genei.dalmago@embrapa.br and mauricio.fernandes@embrapa.br

${ }^{3}$ UNIVATES. Rua Avelino Talini, 171, Bairro Universitário, CEP 95914-014 Lajeado, RS, Brazil. E-mail: schirmbeck.j@gmail.com

${ }^{4}$ Universidade Federal do Rio Grande do Sul, Programa de Pós-Graduação em Sensoriamento Remoto - PPGSR, Centro Estadual de Pesquisa em Sensoriamento Remoto e Meteorologia - CEPSRM. Av. Bento Gonçalves, 9500 - Campus do Vale- Caixa Postal: 15044, CEP 91501-970 Porto Alegre, RS, Brazil.

E-mail: lucimaraws@gmail.com

${ }^{(*)}$ Corresponding author.

\section{ARTICLE INFO}

Article history:

Received 11 September 2019

Accepted 4 March 2020

\section{Index terms:}

global solar radiation

photosynthetically Active Radiation

reflectance

vegetation indices

\section{ABSTRACT}

The objective of this work was to characterize and measure the quantitative and qualitative changes in the solar radiation at the top of the atmosphere as it crosses the earth atmosphere on sunny days and after its interaction with surface cultivated with soybean. The data came from an experiment developed in a soybean crop in the municipality of Carazinho, RS, Brazil, from November 2017 to April 2018. Biophysical, meteorological and radiometric parameters were measured in sunny days, characterizing different stages of development of the soybean cycle. We analyzed the radiation data at the Top of Atmosphere ( $\mathrm{Ra})$, data of global solar radiation (Rg), the Photosynthetically Active Radiation (PAR) and the continuous measurements of the spectral sensors of the incident (i) and reflected ( $\mathrm{r}$ ) in the red (V) and near infrared (IVP) spectrum. From these data the Rg/Ra, PAR/Rg, Vi/IVPi and Vr/IVPr ratios were determined, as well as two vegetation indices. The results have shown that the Earth's atmosphere, as well as surface conditions, modifies the quality and quantity of solar radiation that will be available to plants to grow, and consequently can differentiate regions of food production.

(c) 2020 SBAgro. All rights reserved.

\footnotetext{
CITATION

FONTANA, D. C.; DALMAGO, G. A.; SCHIRMBECK, J.; SCHIRMBECK, L. W.; FERNANDES, J. M. C.

Modificações na quantidade e qualidade da radiação solar ao atravessar a atmosfera e interagir com plantas de soja. Agrometeoros, Passo Fundo, v.27, n.1, p.101-110, set 2019.
} 